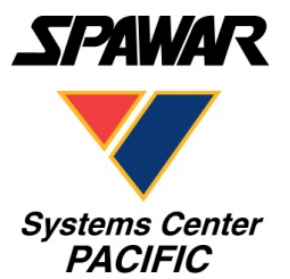

TECHNICAL DOCUMENT 3286

December 2014

\title{
IonRayTrace: An HF Propagation Model for Communications and Radar Applications
}

Stephen Lynch

Approved for public release.

SSC Pacific

San Diego, CA 92152-5001 


\section{SSC Pacific \\ San Diego, California 92152-5001}

\section{ADMINISTRATIVE INFORMATION}

The work described in this report was performed by the Atmospheric Propagation Branch (Code 55280), Space and Naval Warfare Systems Center Pacific (SSC Pacific), San Diego, CA. The Naval Innovative Science and Engineering (NISE) Program at SSC Pacific funded this effort as an Applied Research project.

Released by

D. Tsintikidis, Head

Atmospheric Propagation Branch
Under authority of

P. Juarez, Head

Networks Division

This is a work of the United States Government and therefore is not copyrighted. This work may be copied and disseminated without restriction.

The citation of trade names and names of manufacturers in this report is not to be construed as official government endorsement or approval of commercial products or services referenced in this report.

MATLAB $^{\circledR}$ is a registered trademark of The MathWorks.

Python ${ }^{\circledR}$ is registered trademarks of the Python Software Foundation. 


\section{CONTENTS}

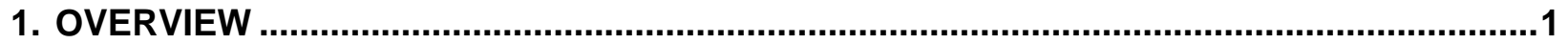

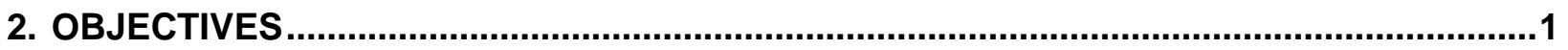

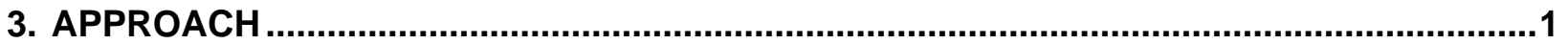

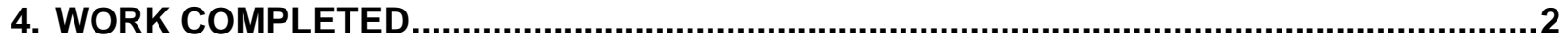

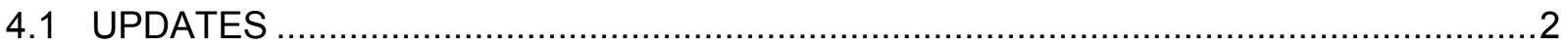

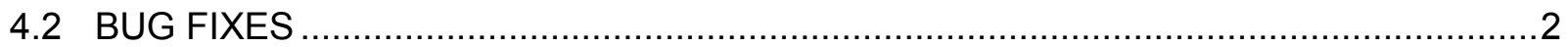

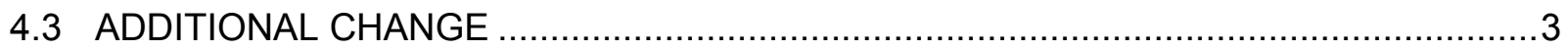

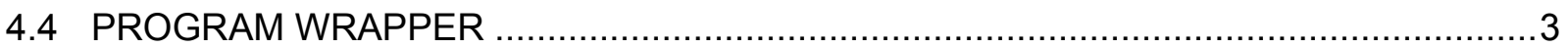

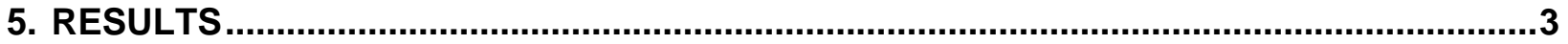

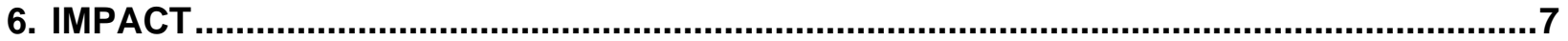

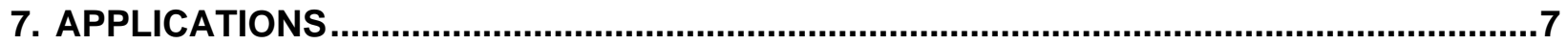

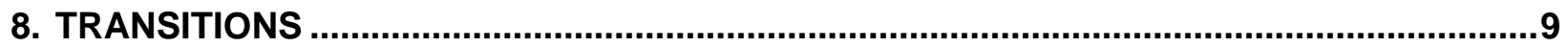

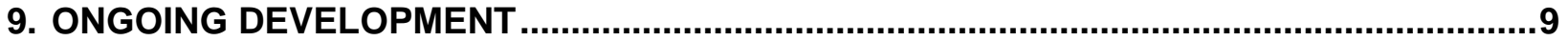

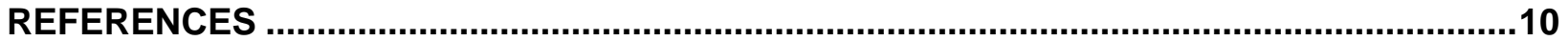

\section{Figures}

1. Great circle path (yellow dashed trace) between a transmitter near SSC Pacific (32.78 N $117.04 \mathrm{~W}$ ) and a point in the Pacific Ocean approximately $2500 \mathrm{~km}$ away bearing $310 \ldots \ldots .4$

2. Critical frequency ( $\mathrm{MHz}$, abscissa) vs. altitude $(\mathrm{km})$ estimated by IRI 2012 with (blue trace)

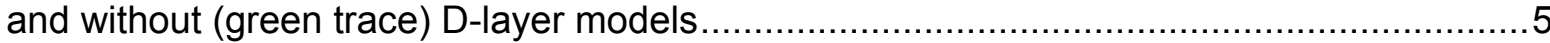

3. Modeled HF rays transmitted from SSC Pacific on 25 May 2014 at 2000 UTC. Take-off elevation angle is $11^{\circ}$ for all rays. Ray color (yellow-orange-red) denotes frequency in $\mathrm{MHz}$, and sky color denotes plasma frequency in $\mathrm{MHz}$

4. Ionospheric absorption $(\mathrm{dB})$ versus frequency $(\mathrm{MHz})$ for each of the ray paths depicted in Figure 3, without (green) and with (blue) D-Region models ...................................... 6

5. Field-of-view map of the Wallops Island, Virginia SuperDARN OTHR .............................. 8

6. Footprint of Wallops Island, Virginia OTHR single-hop illumination at dawn (left) and mid-day (right) on 25 May 2014, with colored traces indicating areas of illumination in the 2- to 7-MHz (red), 7- to 12-MHz (green), and 12- to 17-MHz (blue) bands 


\section{OVERVIEW}

The Advanced Refractivity Effects Prediction System (AREPS) is a tool for planning radio frequency (RF) systems, including communications and $\operatorname{radar}^{1}$. For the purpose of planning highfrequency (HF) communications, a high-fidelity HF propagation model (IonRayTrace) was included in previously distributed implementations of the AREPS suite of propagation estimation tools. However, IonRayTrace was eventually removed from AREPS due to bug-related issues, coupled with a lack of resources (and overall Navy interest) necessary for fixing them. Thus, to maintain minimal HF communications planning ability for use with the HF systems still in the Fleet, a version of the Voice Over America Communications Assessment Program [1], modified for use within AREPS, remained in place of IonRayTrace. Recently renewed interest in high-fidelity HF prediction capabilities now drives an effort to repair and improve the HF propagation model for inclusion in future AREPS distributions, as well as for use in other HF-related applications.

The IonRayTrace model is an implementation of the Jones-Stephens model [2], a threedimensional (3D) Hamiltonian optics-based ray trace program. It uses the International Reference Ionosphere for its environmental background [3]. IonRayTrace's operation is summarized briefly in Section 3. However, the scope of this document is primarily to summarize changes to the model from its previous versions to its current implementation in AREPS, as well as ongoing developments for additional applications.

Sections 2 and 3 summarize the objectives and approach, respectively, of this work. The modifications made to the IonRayTrace source code and operation are discussed in Section 4, and sample results of those changes are presented in Section 5. In Section 6, the probable impacts of this development are discussed. In Section 7, potential applications of an improved IonRayTrace are examined, with sample results. Potential transitions for this work are sketched out in Section 8, and in Section 9 ongoing/additional project efforts (as of the publication date of this document) are summarized. References are provided at the end of this document.

\section{OBJECTIVES}

The overall objective of this work is to produce a high-fidelity HF prediction tool that can be used in diverse HF-based applications with minimal additional development effort. For example, this model should be the basis for long-range HF communications planning, including point-to-point loss, as well as maximum and lowest useable frequency (MUF and LUF) for arbitrary terminal pairs. In addition, IonRayTrace should be used for point-to-area estimation that can inform over-the-horizon radar (OTHR) applications, such as prediction of probabilities of detection at a given time and location.

\section{APPROACH}

The main thrust of this effort involves updating and otherwise improving the IonRayTrace source code, which currently is a mix of FORTRAN77 and FORTRAN90. This effort involves implementing wrappers (e.g., Matlab ${ }^{\circledR}$, Python ${ }^{\circledR}$ ), which enable utilizing IonRayTrace for applications beyond the scope of AREPS. Writing these additional applications enables fast and flexible development, but ultimately they will be implemented as application programming interfaces (APIs) suitable for the platforms on which IonRayTrace is operated (see Section 7).

\footnotetext{
${ }^{1}$ Amalia E. Barrios, Veena Gadwal, and Richard Sprague. 2009. "Modeling RF Digital Signals for Communications Applications." Internal report N0001409AF00002. Please contact the author for further information.
} 
When IonRayTrace is called, either from the command line or within AREPS, it opens and reads an input file that contains information about the date and time, transmitter and receiver locations, frequencies, elevation angles, solar-terrestrial weather (Kp index and 10.7-cm flux), and operation mode. Operation mode currently has four options: homing for point-to-point estimation, rayfan for general assessment of propagation conditions, ionogram for estimating oblique ionograms, and ionosphere model mode, which skips any ray trace calculations and returns ionosphere metrics such as frequency and altitude of the E, F1, and F2 layers. The output is returned in ASCII data files, which may be read using Matlab ${ }^{\circledR}$, Python ${ }^{\circledR}$, or other scripts. When executed within AREPS, these scripts are ingested and plotted by additional AREPS subroutines.

\section{WORK COMPLETED}

IonRayTrace modifications made prior to the next AREPS distribution fall into four categories: (1) updates to the outdated background environmental model (i.e., ionosphere climatology), (2) bug fixes, (3) general changes to the computational flow, and (4) development of a wrapper. The wrapper implements IonRayTrace in basic estimations for calculations specific to OTHR applications. Additional information about these changes is provided in Subsections 4.1 through 4.4. Finally, considerable testing and work was performed to ensure that IonRayTrace functions correctly within the AREPS suite and that the HF model will be included in the next AREPS distribution to the Fleet.

\subsection{UPDATES}

One major update was replacing IRI 2007 with IRI 2012, the latest version. This change involved replacing data files containing climatological coefficients and constants with later versions within the program's directory. In some cases, this update also involved modifications to the source code to account for changes in the background model's data array sizes and formats.

\subsection{BUG FIXES}

Several bugs in IonRayTrace were investigated and fixed.

1. A suspected error in calculation of the azimuthal deviation of the ray path led to the discovery of a conflict between several logical flags associated with the ray's altitude- and range-difference with the receiver, the number of hops (surface reflections), and error states. This conflict affected IonRayTrace's homing and ionogram modes, either by continuing to trace ray paths beyond the data grid or by terminating a ray path computation prematurely. This bug was fixed such that all computation modes (homing, ionogram, rayfan, and ionosphere parameter data) function correctly.

2. A separate error in calculating azimuthal deviation was fixed. Previously, the calculation neglected the sign $( \pm)$ of the ray path's deviations from the vertical plane, resulting only in deviations in the positive $\mathrm{y}$ direction (i.e., to the left if positive $\mathrm{x}$ is in the direction of the receiver).

3. Unnamed common blocks were given names to remove data conflicts, and several data statements were replaced with simple definition statements. Prior to this adjustment, reuse of input parameters from previous IonRayTrace runs within a single AREPS session led to erroneous ray path calculations.

4. Finally, "zombie" code was removed: subroutines that were compiled with the whole solution but were never called were deleted altogether. All of these zombie subroutines had been rendered obsolete, either because they were superseded by more recent and improved versions, or because changes to the overall program flow rendered them unnecessary. 
Note that some of these fixes (1 and 3), while necessary for correct estimation and reinsertion into the next AREPS distribution, are temporary. Use of IonRayTrace for applications such as communications/networking and OTHR will require calculation of additional parameters - and therefore the development of additional modules - such as incident intensity at the receiver or target. Moreover, in some of the more computationally expensive applications, significantly different program structure, including thread-safe execution, will be required to achieve the efficiency necessary for making useful predictions or estimates within strict time requirements (see Section 9).

\subsection{ADDITIONAL CHANGE}

Aside from repairing broken code, a major change was made to the way the background environment is computed: D-Region (also called the D-layer) models were re-implemented. The D-Region, which occurs in the lowermost $40 \mathrm{~km}$ of the ionosphere (60- to $100-\mathrm{km}$ altitude) on the dayside of the globe, typically contains a relatively low density of free electrons $\left(10^{2}\right.$ to $\left.10^{4} \mathrm{~cm}^{-3}\right)$ and has negligible refractive effect on electromagnetic waves in the HF band [5]. However, in general, the rates of collisions of free electrons with neutral particles are much higher in the D-Region, leading to significant absorption of HF waves. Because accurate estimation of absorption losses is critical for both communications (e.g., link budget) and radar-related (e.g., probability of detection) problems, this is an important capability for the HF prediction tool.

For the D-layer models to have an impact on IonRayTrace's output, the vertical gridding of the electron density values returned by IonRayTrace also had to be changed to include lower altitude values, as the lowest value previously returned was $90 \mathrm{~km}$. Thus the output grid now includes finer (5-km spacing) gridding in lower (down to 65-km) altitudes in exchange for coarser (up to $20 \mathrm{~km}$ from 10-km step size) sampling of the uppermost $(900-$ to $1000-\mathrm{km})$ portion of the ionosphere. The total number of grid points was left unchanged. While this trade-off does not affect the actual ray trace computation (the output grid is a subset of the electron density data computed for the ray trace algorithm), it is an acceptable trade-off, as none of the HF systems of interest will be directly affected by the ionosphere's topside. Uncertainty of the total electron content (TEC) estimates derived from the IRI data may be affected by this change.

Subsequently, the maximum number of steps per hop in the ray trace computation also had to be increased considerably, as the Runge-Kutta/Adams-Moulton Ordinary Differential Equation (ODE) solver [2] utilizes very small step sizes in this lower region, leading in turn to premature termination of the ray trace as the maximum number of steps was reached. The ultimate result was that no rays were found for even simple scenarios with relatively short ranges between transmitter and receiver.

\subsection{PROGRAM WRAPPER}

A Matlab ${ }^{\circledR}$-based OTHR-specific wrapper (MOTHR) was developed that enables using IonRayTrace for basic prediction of OTHR function. MOTHR returns overall footprints of the OTHR's effective field of view, based on ionospheric conditions predicted for a given time, and the location and technical specifications (minimum and maximum detection ranges, minimum and maximum azimuthal angles) of the OTHR system of interest.

\section{RESULTS}

To compare the effects of IonRayTrace modifications to those of the previous (most) stable version, several test cases were run. One test cases is detailed in this document, a link modeled between a surface-based transmitter near SSC Pacific $(32.78 \mathrm{~N} 117.04 \mathrm{~W})$ and a surface-based receiver approximately $2500 \mathrm{~km}$ away in the Pacific Ocean at a bearing of $310^{\circ}(42.7 \mathrm{~N}, 141.15 \mathrm{~W})$ on 25 May 2014 at 2000 UTC. See Figure 1. 


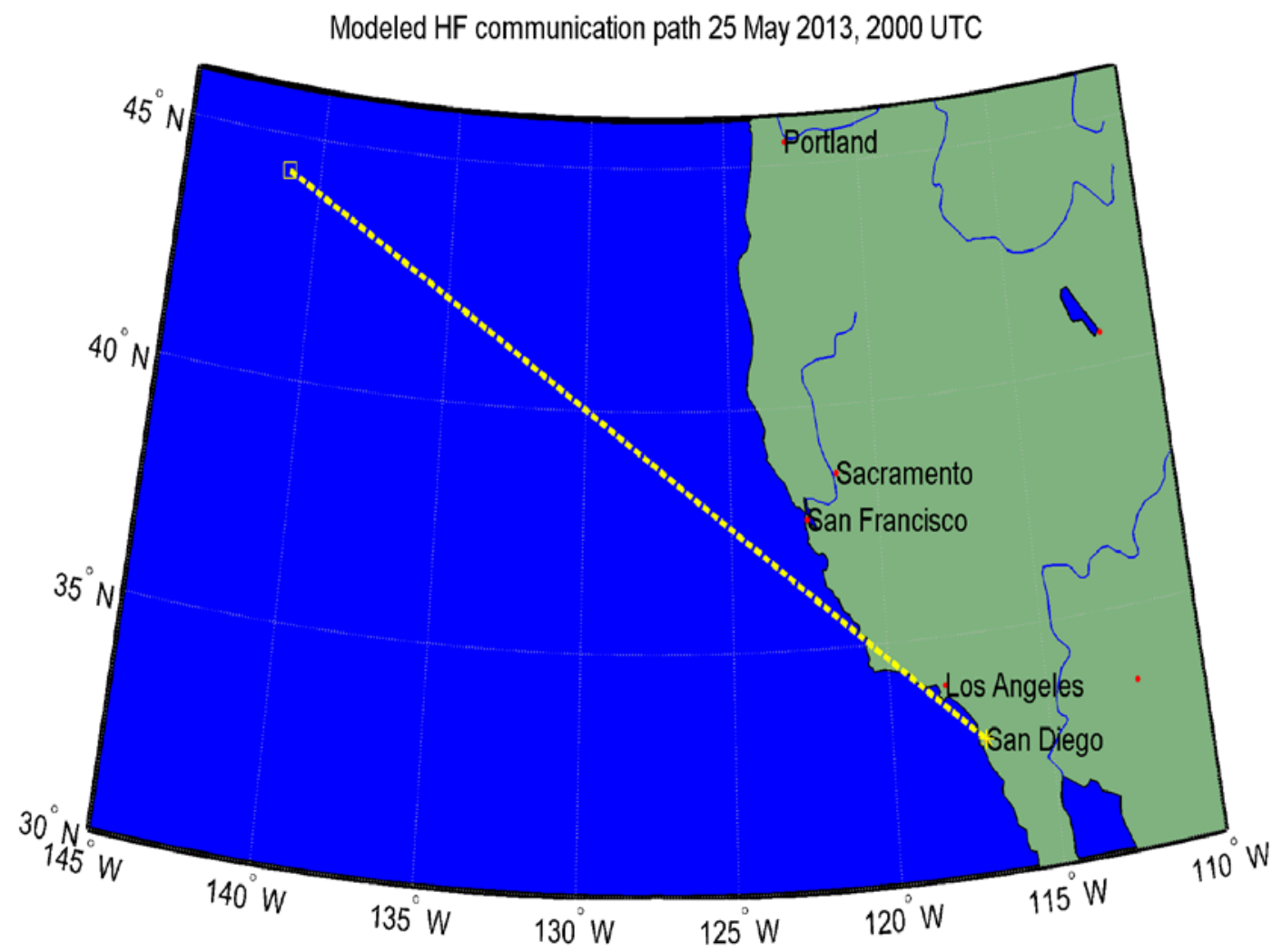

Figure 1. Great circle path (yellow dashed trace) between a transmitter near SSC Pacific (32.78 N $117.04 \mathrm{~W}$ ) and a point in the Pacific Ocean approximately $2500 \mathrm{~km}$ away bearing 310.

The resonant plasma frequency $f_{o}(z)$ in the ionosphere (i.e., the critical frequency for a vertically incident electromagnetic wave) is

$$
f_{o}(z)=\frac{e_{-}}{2 \pi} \sqrt{\frac{N_{e_{-}}(z)}{m_{e_{-}} \epsilon_{o}}},
$$

where $N_{e_{-}}$is the density of free electrons at altitude z; $e_{-}$and $m_{e_{-}}$are the charge and mass, respectively, of an electron; and $\epsilon_{o}$ is the permittivity of free space [5]. A plot of vertical profiles of plasma frequency $f_{o}(z)$ with and without the D-Region components immediately points to several potential differences in IonRayTrace's results. Figure 2 shows clearly that the D-Region contribution to the overall electron density, as estimated by IRI 2012, reaches up above the nominal D-region altitudes of 60 to $100 \mathrm{~km}$, resulting in higher densities at the E-Region $(\sim 110 \mathrm{~km})$ and low F1 $(110$ to $130 \mathrm{~km})$. This effect is probably an artifact resulting from IRI effectively integrating those electron density contributions that would be D-Region into higher altitudes when the height distribution models are turned off. It is not currently known why this upward shift occurs, but this artifact has the potential to distort IonRayTrace's HF propagation predictions. 


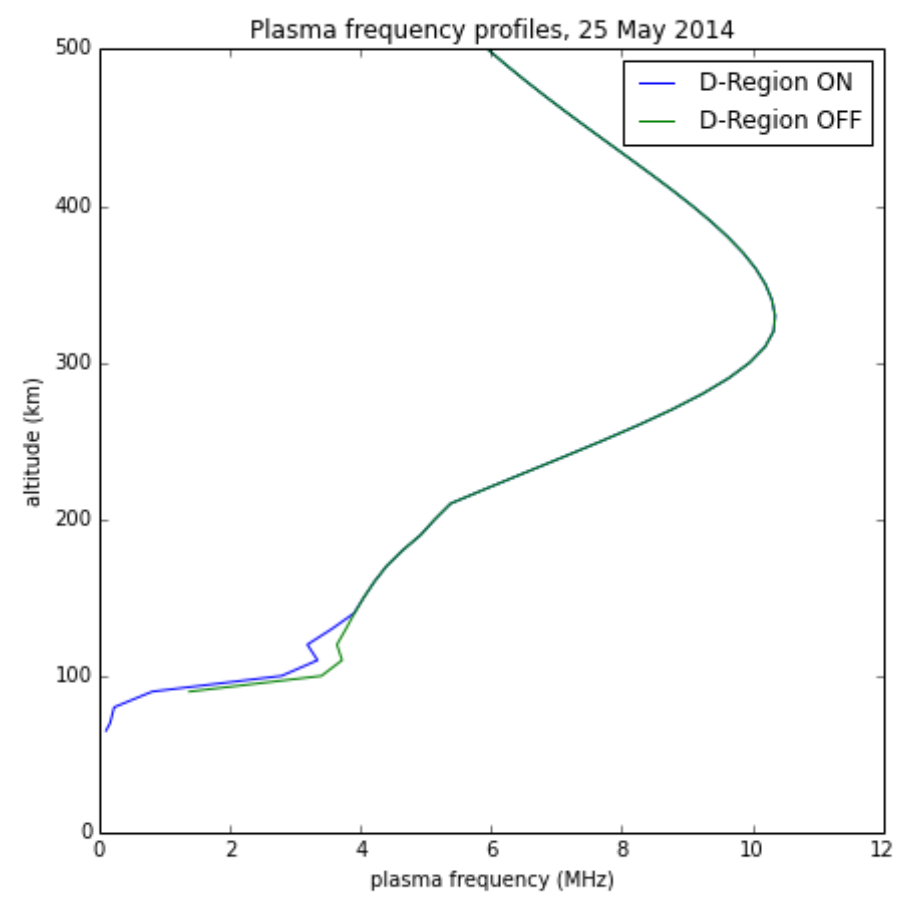

Figure 2. Critical frequency ( $\mathrm{MHz}$, abscissa) vs. altitude $(\mathrm{km})$ estimated by IRI 2012 with (blue trace) and without (green trace) D-layer models.

This altered density structure in the lower portion of the ionosphere model significantly influences the ray path calculations. Figure 3 illustrates this clearly; the two panes show results from the IonRayTrace with all inputs and algorithms identical except the inclusion of D-Region models. In both cases, rays with frequencies ranging from 8.0 to $25.0 \mathrm{MHz}$ were launched, all with an initial elevation angle of $11^{\circ}$, and were propagated to a distance of roughly $2500 \mathrm{~km}$. In the previous implementation of IonRayTrace, with the D-Region models turned off, the ray paths computed by the model are nearly independent of frequency (Figure 3, top). In contrast, with the D-Region models activated, there is a distinct frequency dependence to the resulting paths, which affects the maximum altitude, and the number of hops along the path.

Adding the D-Layer models also affects the estimated absorption values - this was the primary anticipated consequence of this change. While Figure 4 illustrates that the D-Region models and resulting free electron density and collision rate estimates strongly affect the absorption estimates, additional information is needed to clearly illustrate the complex relation between signal frequency, maximum ray altitude, ray phase and group path lengths, number of hops, and collision frequency. 

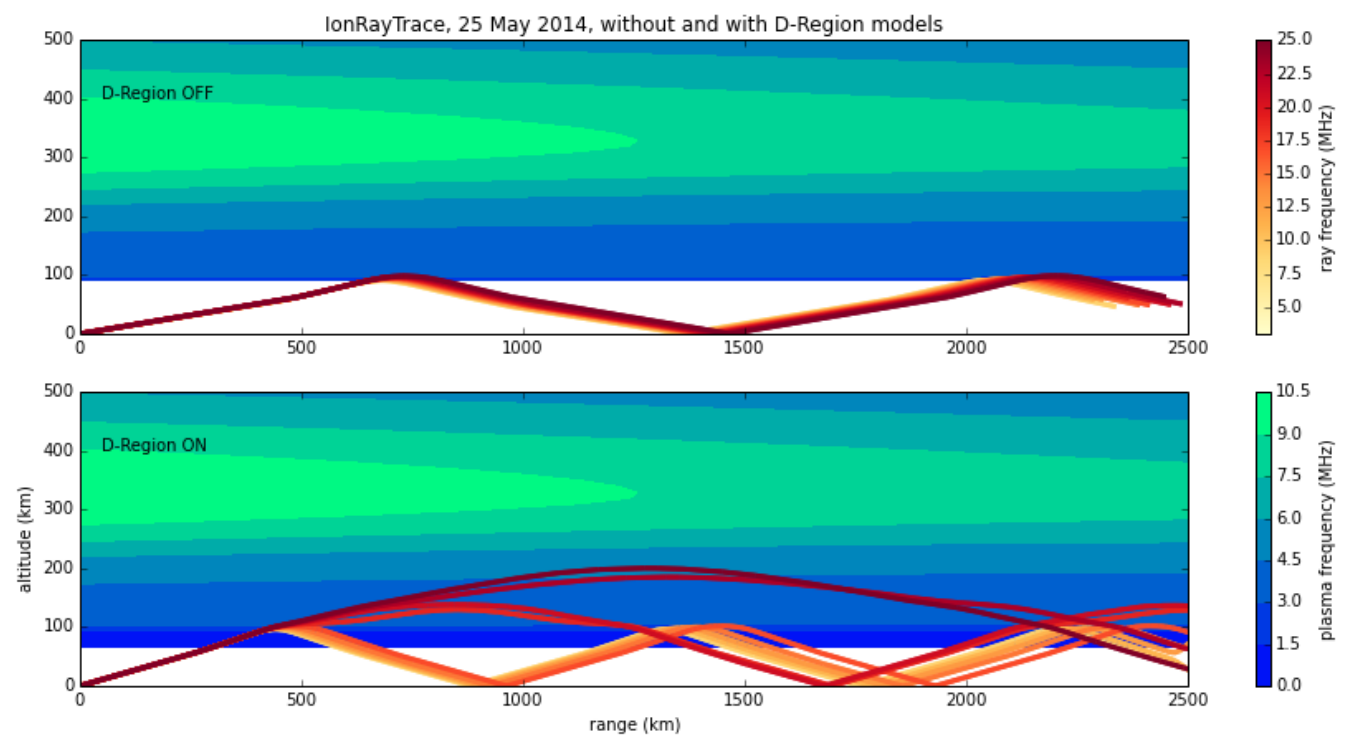

Figure 3. Modeled HF rays transmitted from SSC Pacific on 25 May 2014 at 2000 UTC. Take-off elevation angle is $11^{\circ}$ for all rays. Ray color (yellow-orange-red) denotes frequency in $\mathrm{MHz}$, and sky color denotes plasma frequency in $\mathrm{MHz}$.

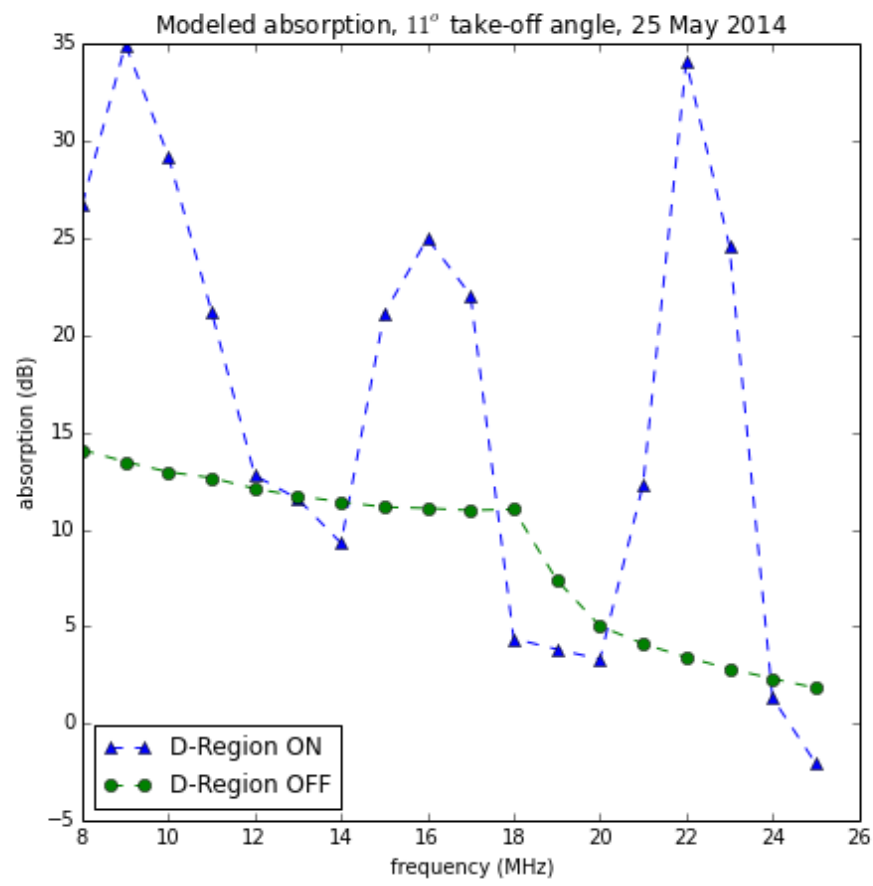

Figure 4. Ionospheric absorption $(\mathrm{dB})$ versus frequency $(\mathrm{MHz})$ for each of the ray paths depicted in Figure 3, without (green) and with (blue) D-Region models. 


\section{IMPACT}

An increase in the low-altitude estimated densities (Figure 2) results in stronger refraction and reflection at and just above the E-Layer, leading in turn to an artificial increase in paths supported, as well as a reduction in the range of a single hop, especially at HF frequencies up to the E-layer critical frequency (Figure 3). This latter effect is especially true for paths that penetrate the E-layer but whose frequency is not sufficiently high to reach the upper F1 or F2 layers; their predicted group and phase path lengths will be distorted by the artificial increase in estimated electron densities in the lower F1 layer. This effect will be especially prevalent in the daytime, as the D-Region is a dayside phenomenon.

These improvements will greatly benefit system-specific information derived from the HF propagation model IonRayTrace. For instance, probability of detection by OTHR is driven by several factors, including intensity at the target at the operating frequency [6]. Moreover, OTHR generally operates at a single frequency at a time, and that frequency is chosen to investigate at and near a particular range from the transmitter. Thus, it is critical that an OTHR prediction tool accurately predict these illumination ranges for reliable assessment of OTHR capability.

Similarly, accurate predictions of HF signal intensity and ray distance are vital for reliable communications plans. Errors could lead either to erroneous predictions of which frequencies are supported by the propagation conditions and are therefore suitable for links between given node pairs, or even the reciprocal error, by missing potential frequencies in the communications plan. Both these errors would be hugely detrimental to any HF-based networking effort [7].

Enabling computation of electron density estimates in the D-Layers also required modification to the vertical gridding; lower minimum altitudes and finer grid spacing in the lowest $50 \mathrm{~km}$ was enabled in exchange for coarser gridding at the uppermost altitudes. While this change should have no effect on bottomside applications (applications where HF signals are confined to the underside of the ionosphere), it may lead to greater errors in estimating of total electron content along paths penetrating the ionosphere. Thus, caution must be exercised if TEC is to be incorporated in any environment update algorithm.

\section{APPLICATIONS}

An undeveloped application for this modeling capability is communications planning for a moving platform such as an HF-equipped ship. As propagation conditions change due to the daily variability of the ionosphere, so too must radio channels, if links are to be maintained. Motion of the terminal across the globe only complicates the problem further. An easily executed, or even automated, communications-specific API will streamline this process and even enable more sophisticated communications plans, including flexible networks.

Another area where IonRayTrace's propagation predictions will be useful is in assessment of OTHR performance [6]. MOTHR creates input files based on the OTHR's specifications (e.g., azimuthal resolution and span, minimum and maximum ranges), executes IonRayTrace and collects the output to estimate HF signal intensity incident within the OTHR receiver array's field of view (FOV). From IonRayTrace's output of ray fans that are evenly spaced in the vertical (to mimic effective isotropic radiated power [EIRP]), MOTHR estimates intensity over the entire gridded FOV by counting the incident rays in each spatial bin. While this is a rather crude estimate of field intensity, it has been shown to work reasonably well in another application [4]. Moreover, a module estimating the intensity based on transmitter power and manifold, as well as ray path metrics (spatial derivatives), is currently under development (see Section 9). In addition, MOTHR filters the incident 
rays within each gridded bin, keeping only those incident rays whose hop count is below a userspecified cut-off. Finally, MOTHR organizes the rays into separate subbands within the HF spectrum.

To demonstrate this utility, a sample case was created using the technical specifications of the Super Dual Auroral Radar Network (SuperDARN) OTHR located at the NASA Flight Facility at Wallops Island, Virginia. The SuperDARN's band-separated footprint and overall (incoherently summed) intensity incident at the surface over the combined footprint were estimated for night and day conditions on 25 May 2014.

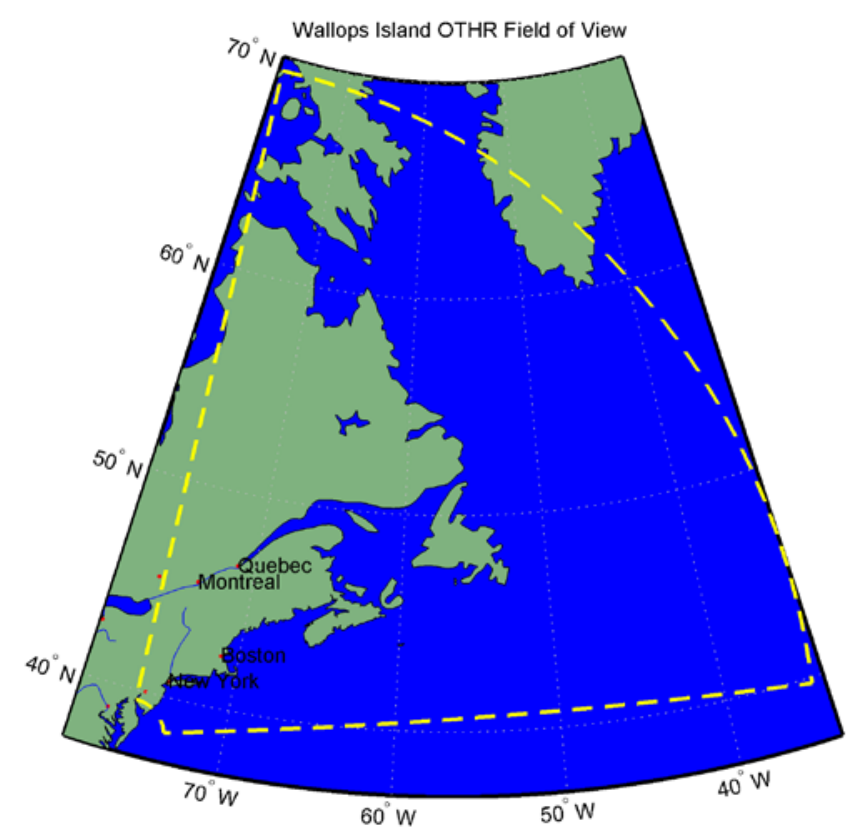

Figure 5. Field-of-view map of the Wallops Island, Virginia SuperDARN OTHR.

Figure 6 shows clear differences in incident intensity and illuminated footprint for each subband. In general, lower incident intensity can be expected at night and through dawn, as the lower free electron densities result in lower critical frequencies. Thus, waves over more of the HF spectrum escape to space through the ionosphere when emitted at night. In addition, at night and at the highest frequency band, the innermost range of illumination increases and the longest detection range in general decreases, though not at all azimuths. In contrast, there is little difference in minimum illumination ranges across the three bands during mid-day. 

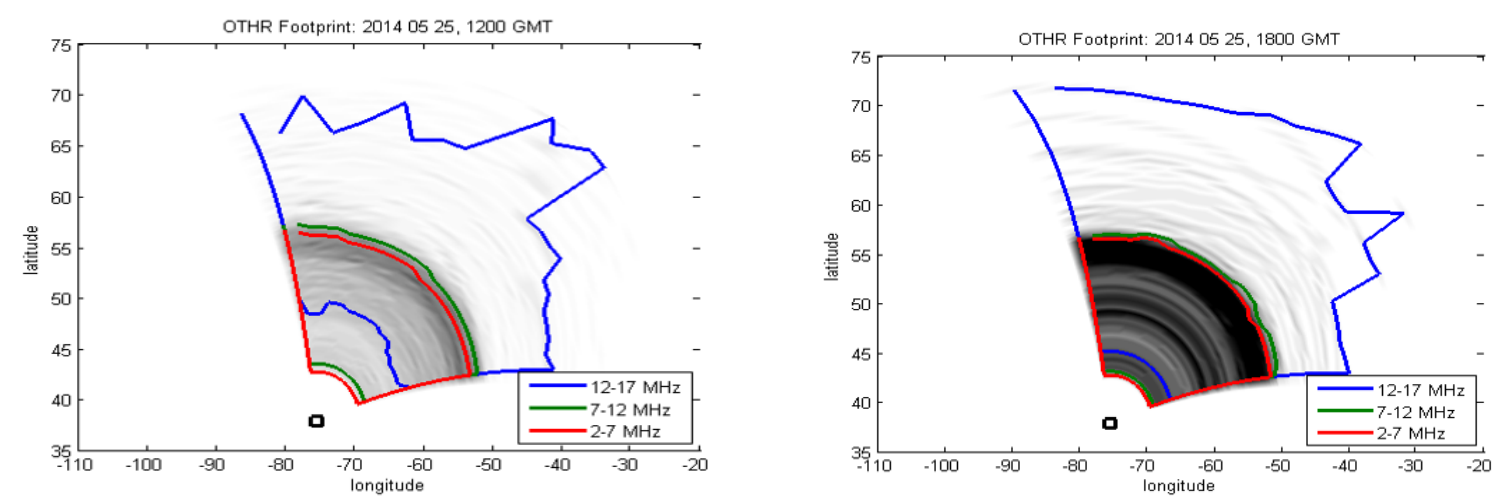

Figure 6. Footprint of Wallops Island, Virginia OTHR single-hop illumination at dawn (left) and midday (right) on 25 May 2014, with colored traces indicating areas of illumination in the 2- to 7-MHz (red), 7- to 12-MHz (green), and 12- to 17-MHz (blue) bands.

\section{TRANSITIONS}

Beyond reinsertion of the high-fidelity HF ray trace code into future AREPS distributions, there is considerable interest in adding IonRayTrace either whole cloth or as separate modules into the next generation suite of afloat RF prediction tools. Given the overall interest in diverse network architecttures, there is also considerable interest in including IonRayTrace's modules in a near-real-time communications network planning tool. Finally, there is great potential in an accurate and computationally efficient propagation model to perform environmental inversion from diverse data inputs. This latter possibility could create numerous opportunities in studying ionospheric dynamics using basic HF communications systems, for example.

\section{ONGOING DEVELOPMENT}

Ongoing project efforts include development of an HF channel estimation model that incorporates the spatial and temporal variability of the ionosphere. Such a channel model will require accurate estimates of signal intensity, environmentally driven amplitude and phase variations, as well as surface reflection and scattering effects. For communications-related applications, models of the different sources of environmental variation on data and error rates are necessary for reliable communications plans. Similarly, OTHR system-specific models are required for modeling the impact of ionosphere variability on detection algorithms.

Modification of IonRayTrace's source code to include flexible gridding and arbitrary node pair counts will enable it both for communications planning along long distance routes, and for broad networks. Additional gains can be made using the above system modeling applications if IonRayTrace can be implemented in a data assimilation scheme that updates propagation conditions based on realized signals. However, all of these capabilities will require substantial restructuring of IonRayTrace's source code, including making the code more modular, such that the environmental parameters can be separated and perturbed, as well as modifying the entire program to be thread-safe (to account for the computationally burdensome inversion problems associated with data assimilation).

Finally, testing of this program's parts and overall function is warranted. Current and future work includes field campaigns and model-data comparison. 


\section{REFERENCES}

1. G. Lane. 2001. "Signal-to-Noise Predictions Using VOACAP, Including VOAAREA." Rockwell Collins, Cedar Rapids, IA.

2. J. A. Ferguson and C. H. Shellman. 1993. "The High-frequency Benchmark Propagation Program.” NRaD (now SSC Pacific) Technical Document 2608. San Diego, CA.

3. D. Bilitza, L.-A. McKinnell, B. Reinisch, and T. Fuller-Rowell. 2010. "The International Reference Ionosphere Today and in the Future," Journal of Geodesy, doi:10.1007/s00190010-0427-x.

4. A. Barrios. 2004. "Estimation of Surface-based Duct Parameters from Surface Clutter using a Ray Trace Approach,” Radio Science, doi:10.1029/2003RS002930.

5. J. M. Kelso. 1959. Radio Ray Propagation. McGraw-Hill Book Company, New York, NY.

6. S. V. Fridman and L. J. Nickisch. 2001. "Generalization of Ionospheric Tomography on Diverse Data Sources: Reconstruction of the Three-dimensional Ionosphere from Simultaneous Vertical Ionogams, Backscatter Ionograms, and Total Electron Content Data," Radio Science, doi:10.1029/1999RS002405/

7. L. J. Nickisch, G. St. John, S. V. Fridman, M. A. Hausman, and C. J. Coleman. 2012. "HiCIRF: A High-fidelity HF Channel Simulation," Radio Science, vol. 47, no. 4, doi:10.1029/2011RS004928. 


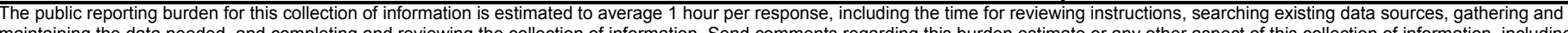

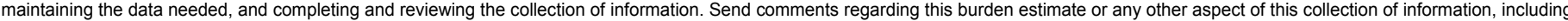

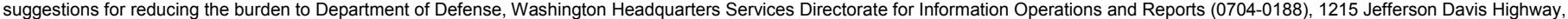

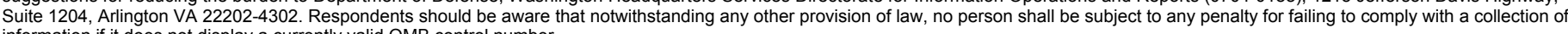

PLEASE DO NOT RETURN YOUR FORM TO THE ABOVE ADDRESS.

\section{REPORT DATE (DD-MM-YYYY) \\ December 2014 \\ Final}

4. TITLE AND SUBTITLE

\section{TITLE AND SUBTITLE}

IonRayTrace: An HF Propagation Model for Communications and Radar Applications

IonRayTrace: An HF Propagation Model for Communications and Radar Applications

\section{AUTHORS}

Stephen Lynch

7. PERFORMING ORGANIZATION NAME(S) AND ADDRESS(ES)

SSC Pacific, 53560 Hull Street, San Diego, CA 92152-5001

9. SPONSORING/MONITORING AGENCY NAME(S) AND ADDRESS(ES)

Naval Innovative Science and Engineering (NISE) Program (Applied Research)

SSC Pacific, 53560 Hull Street, San Diego, CA 92152-5001

\section{DISTRIBUTION/AVAILABILITY STATEMENT}

Approved for public release.

\section{SUPPLEMENTARY NOTES}

This is work of the United States Government and therefore is not copyrighted. This work may be copied and disseminated without restriction.

\section{ABSTRACT}

The overall objective of this work is to produce a high-fidelity, high-frequency (HF) prediction tool that can be used in diverse HF-based applications with minimal additional development effort. For example, this model should be the basis for long-range HF communications planning, including point-to-point loss, as well as maximum and lowest useable frequency (MUF and LUF) for arbitrary terminal pairs. In addition, IonRayTrace should be used for point-to-area estimation that can inform over-the-horizon radar (OTHR) applications such as prediction of probabilities of detection at a given time and location.

\section{SUBJECT TERMS}

Mission Area: Communications

radio frequency high-frequency communications over-the-horizon radar Advanced Refractivity Effects Prediction System

16. SECURITY CLASSIFICATION OF:

\begin{tabular}{|c|c|c|}
\hline a. REPORT & b. ABSTRACT & c. THIS PAGE \\
$\mathrm{U}$ & $\mathrm{U}$ & $\mathrm{U}$ \\
\hline
\end{tabular}

\section{LIMITATION OF ABSTRACT}

$\mathrm{U}$

18. NUMBER
OF
PAGES

3. DATES COVERED (From - To)

5a. CONTRACT NUMBER

5b. GRANT NUMBER

5c. PROGRAM ELEMENT NUMBER

5d. PROJECT NUMBER

5e. TASK NUMBER

5f. WORK UNIT NUMBER

8. PERFORMING ORGANIZATION REPORT NUMBER

TD 3286

11. SPONSOR/MONITOR'S REPORT NUMBER(S) 


\section{INITIAL DISTRIBUTION}

$84300 \quad$ Library

$85300 \quad$ Archive/Stock

(1)

55280

S. Lynch

(1)

Defense Technical Information Center

Fort Belvoir, VA 22060-6218

(1) 
Approved for public release.

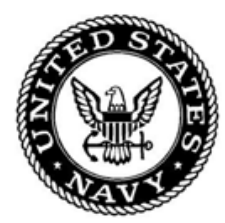

SSC Pacific San Diego, CA 92152-5001 